\title{
Mechanische Beatmung in der Neonatologie
}

Ulrich H. Thome

\section{Übersicht}

Pathophysiologische Grundlagen

Lungenprotektive Beatmung

Optimale Beatmungseinstellungen

finden

Patientengesteuerte

Beatmungsmodi

Nichtinvasive Beatmung

Zusammenfassung

\section{5}

338

339

341

345

347

\section{Pathophysiologische Grundlagen}

Die mechanische Beatmung eines Neugeborenen und insbesondere eines kleinen Frühgeborenen stellt eine besondere Herausforderung dar. Besonders im kranken Zustand, d.h. im Rahmen des Atemnotsyndroms, verträgt die neonatale Lunge nur kleine Tidalvolumina (Abb. 1). Große Tidalvolumina sind aufgrund der geringen Compliance schwer erreichbar und außerdem schädlich. Aufgrund der lungenmechanisch extremen Situation müssen bei schweren Erkrankungen oftmals die Grenzen der Physik ausgeschöpft werden.

Man geht in Analogie zu Studien an erwachsenen Intensivpatienten [2] davon aus, dass Tidalvolumina von $4-6 \mathrm{ml} / \mathrm{kg}$ Körpergewicht optimal sind.

Cave: Je höher die Tidalvolumina, umso schneller entstehen beatmungsbedingte Schäden.

Um eine ausreichende Ventilation (Atemminutenvolumen) zu erreichen, sind daher zur Kompensation der kleinen Tidalvolumina relativ hohe Beatmungsfrequenzen erforderlich. Hohe Frequenzen können nur effektiv sein, wenn das Beatmungsgerät zu schnellen Druckänderungen im Beatmungsschlauchsystem in der Lage ist. Hierfür ist eine Minimierung des kompressiblen Volumens erforderlich, was durch Verwendung kleiner Schlauchdurchmesser und kleiner Befeuchter-

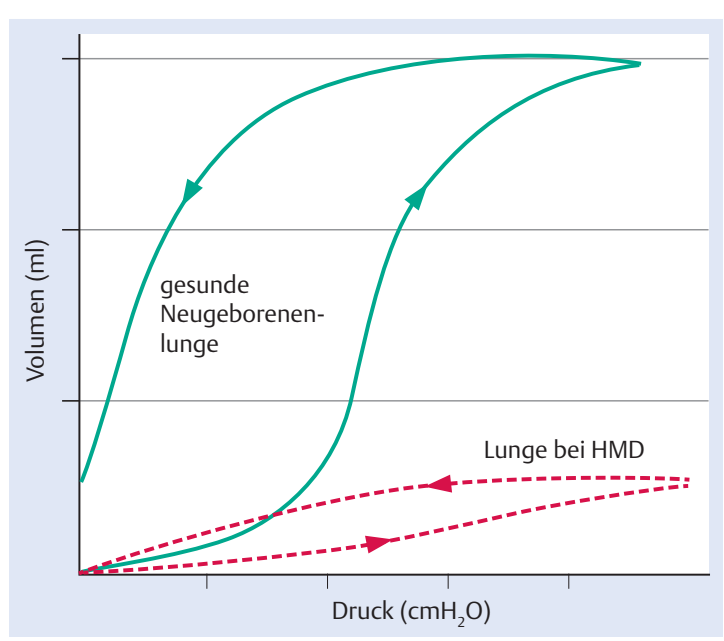

Abb. 1 DruckVolumen-Kurve bei Surfactantmangel im Vergleich zu normaler Lungenfunktion (HMD: hyalines Membransyndrom) [1].

töpfe erreicht wird. Außerdem muss die pneumatische Technik mit entsprechender Geschwindigkeit arbeiten können.

\section{Merke: Eine schonende Beatmung besteht aus kleinen Tidalvolumina. Die Frequenz muss kom- pensatorisch hoch und die verwendete Technik dementsprechend schnell sein.}

Abgesehen von der durch technische Maßnahmen optimierbaren Beatmungsgerätetechnik gibt es für die maximal mögliche Beatmungsfrequenz auch biologisch-physikalische Grenzen. Diese hängen von den 
mechanischen Eigenschaften der Lunge des jeweiligen Patienten und somit vom Erkrankungsstadium sowie vom Tubusdurchmesser ab. Werden Inspirationszeit oder Exspirationszeit zu stark verkürzt, sind die Atemphasen unvollständig und es kommt bei zu kurzer Exspirationszeit zu unvollständiger Exspiration und gefangener Luft (Air Trapping) in der Lunge. Diese Grenze kann nur mit speziellen Verfahren (Hochfrequenzbeatmung) überschritten werden.

Außerdem steigt mit sinkendem Tidalvolumen der relative Anteil der Atemluft, die im Totraum verbleibt und den Alveolarraum nicht erreicht. Mit Tidalvolumina, die dem Totraum entsprechen, ist zumindest theoretisch kein Gasaustausch mehr möglich, sodass das Tidalvolumen immer deutlich größer als der Totraum sein muss. Diese Grenze kann nur mit Hochfrequenzbeatmung unterschritten werden.

Merke: Für die normale Beatmung ergibt sich daraus, dass es vorteilhaft ist, den durch die erforderlichen Gerätschaften zusätzlich verursachten Totraum möglichst gering zu halten.

Eine weitere Schwierigkeit entsteht durch die oftmals geringe Kooperation der Patienten. Frühgeborene passen sich oft nicht dem Rhythmus des Beatmungsgeräts an und aufgrund ihrer unregelmäßigen Atmung ist es auch oftmals nicht gut möglich, eine starre Beatmungsfrequenz optimal an das Frühgeborene anzupassen. Für die vom Patienten zwischendurch ausgeführten Atemzüge muss das Beatmungsgerät jedoch jederzeit dem Patienten ausreichend Luft anbieten, da ein Inspirationsversuch gegen geschlossene Ventile nicht nur äußerst unangenehm ist, sondern auch zu einem Verlust des Druckes und zur vermehrten Bildung von Atelektasen beitragen könnte. Außerdem können Versuche des Kindes, zu schreien, in der Bildung von Atelektasen enden, da das Kind dabei kräftig ausatmet, aber aufgrund des liegenden Endotrachealtubus nicht gleichzeitig die Stimmritze schließen kann, sodass unwillkürlich eine viel zu große Menge Luft aus der Lunge entweicht [3].

Ein weiterer wichtiger Punkt bei der Beatmung ist die Befeuchtung. Unzureichend angefeuchtete Atemluft kann durch Verdunstungskälte dem Kind in den Atemwegen sehr viel Wärme entziehen. Außerdem kommt es durch Eintrocknung von Sekret in den relativ engen Endotrachealtuben sehr rasch zu einer Tubusobstruktion. Andererseits kann eine übermäßige Anfeuchtung der Atemluft durch Kondenswasserbildung und dessen rezidivierenden Aspirationen die kindliche Atmung deutlich destabilisieren. Schließlich ist es wichtig, dass der Befeuchter einen möglichst geringen Totraum (=kompressibles Volumen) aufweist, um eine rasch ansprechende Druckregulation durch das Beatmungsgerät zu ermöglichen.

\section{Technische Entwicklungen}

Die erste technische Lösung, die den Anforderungen der Neonatalbeatmung tatsächlich gerecht werden konnte, war das zeitgesteuerte druckbegrenzte Beatmungsgerät mit konstantem Fluss im Beatmungsschlauchsystem. Durch den konstanten Fluss im Schlauchsystem stand immer genügend Luft zur Verfügung, sodass das Kind jederzeit nach Belieben einen Atemzug einfügen konnte (Abb.2).

Auch die heutigen modernen Geräte lassen ihre Abstammung von den druckbegrenzten zeitgesteuerten Konstantfluss-Beatmungsgeräten deutlich erkennen. Sie verfügen allerdings i.d.R. über eine automatische Flussregelung, die es den Steuerungsrechnern ermöglicht, den Fluss bedarfsgerecht entsprechend den Atemphasen zu variieren, wenngleich auch weiterhin während der Exspiration ein erheblicher Fluss im Schlauchsystem aufrecht erhalten wird. Außerdem wurden verschiedene Techniken eingeführt, um die Atembemühungen des Patienten zu erkennen und für die Steuerung der Beatmung nutzbar zu machen.

Messtechniken zur Erkennung patienteneigener Atembemühungen lassen sich in verschiedener Weise zur Steuerung des Beatmungsgeräts einsetzen. Soll nur der Beginn der patienteneigenen Inspiration festgestellt und dadurch eine Reaktion des Beatmungsgeräts ausgelöst werden, spricht man von Triggerung oder von getriggerter Beatmung. Darüber hinaus ist es denkbar, auch das Ende der patienteneigenen Inspiration zu erfassen und davon abhängig die maschinelle Inspiration abzubrechen, was dem Patienten auch die Kontrolle über seine Inspirationszeit zurückgibt. Man spricht von „pressure support“ oder druckunterstützter Beatmung. In einem weiteren Schritt kann die Atemanstrengung des Patienten über den gesamten Atemzyklus in Echtzeit erfasst und dem Patienten eine Unterstützung proportional zu seinen eigenen Atemanstrengungen angeboten werden. Dies bezeichnet man als proportional assistierende Beatmung.

Die am häufigsten eingesetzte Messmethode ist der Inline-Flowsensor, trotz seiner nachteiligen Auswirkungen auf den Totraum, weil er die meiste Information 
liefert und somit auch die größte Palette an assistierenden Beatmungsverfahren ermöglicht. Am häufigsten verwendet werden Pneumotachografen oder Hitzdraht-Anemometer. Ultraschall-Flowmeter spielen keine bedeutende Rolle. Pneumotachografen sind genauer, aber anfälliger für Kondenswasser. HitzdrahtAnemometer messen niedrige Flüsse schlechter, sind dadurch ungenauer und können ohne zusätzlichen konstruktiven Aufwand die Strömungsrichtung nicht erkennen. Außerdem sind die dünnen Drähte mechanisch und für Korrosion empfindlich.

Der Flowsensor eignet sich zur Feststellung des Beginns einer vom Patienten ausgehenden Inspiration ebenso wie zur Festlegung des vom Patienten gewünschten Inspirations- und Exspirationsendes, sodass mit einem Flowsensor getriggerte und druckunterstützte Beatmungsmodi möglich sind. Außerdem kann der Flowsensor anhand der Stärke des Inspirationsflusses und durch Integration des Flowsignals das inspirierte Volumen ermitteln, auf Wunsch auch die Beatmung danach steuern und ermöglicht so die Volumengarantie und die proportional assistierende Beatmung, bei der der Patient die volle Kontrolle über seine Atmung erhält.

Merke: Mit einem Flowsensor sind getriggerte und druckunterstützte Beatmungsmodi möglich.

Es gibt keinerlei klinische Studien, die bewiesen haben, dass der Einsatz eines Flowsensors und die Kenntnis des Tidalvolumens als solche die Behandlungsergebnisse verbessern. Am Ende muss der Arzt entscheiden, ob der durch die Messtechnik erzielte Informationsgewinn oder die Möglichkeit, die Beatmung mit der Eigenatmung zu synchronisieren oder neue Beatmungsmodi einzusetzen, tatsächlich die höheren „Kosten " für den Patienten in Form der Notwendigkeit eines höheren Tidalvolumens aufwiegen, zumal die meisten der neueren Beatmungsmodi noch nicht in randomisierten Studien geprüft worden sind und somit der Nutzen nicht als erwiesen angesehen werden kann.

Merke: Der Arzt sollte genaue Kenntnisse über die Für und Wider der Beatmungstechniken besitzen, um eine kompetente Behandlungsentscheidung treffen zu können.

Aufgrund des besonders für sehr kleine Kinder nachteiligen Flowsensor-Totraums wurden auch andere Methoden zur patientengesteuerten Beatmung entwickelt. Alle diese Methoden beschränken sich jedoch auf die Triggerung, d.h. auf das möglichst schnelle Erkennen des Beginns einer Inspiration (Abb.3).

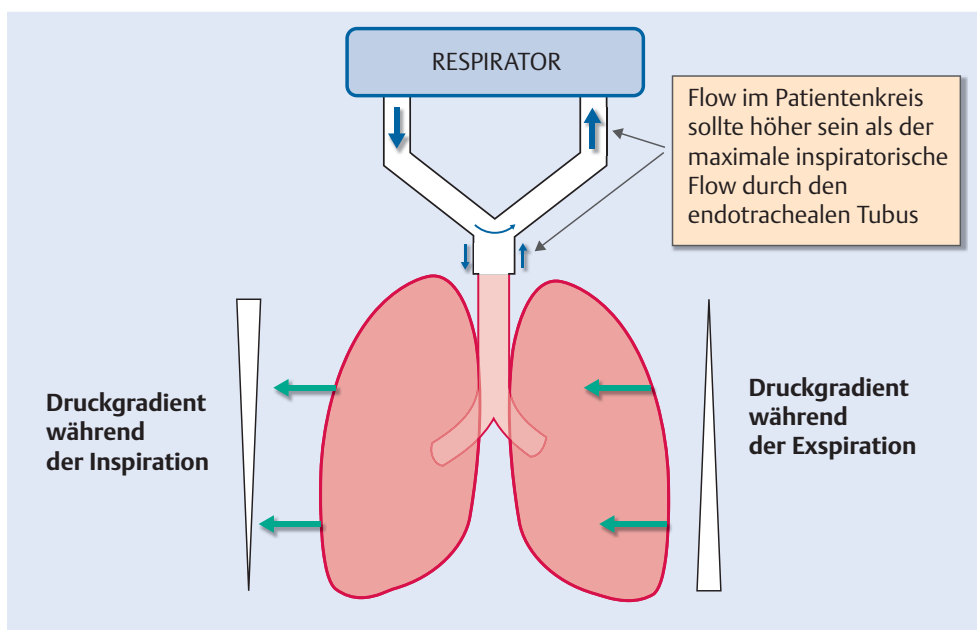

Abb.2 Funktionsweise eines Neonatalbeatmungsgeräts.

\section{Auswirkung einer Vergrößerung des Totraums}

Ein Zielkonflikt entsteht durch den Totraum, den zwangsläufig jedes in den Atemweg eingebaute Messinstrument mit sich bringt. Jedes Einbauteil, das den Totraum vergrößert, wird die $\mathrm{CO}_{2}$-Auswaschung verschlechtern, was über ein größeres Tidalvolumen mit einer erhöhten beatmungsbedingten Lungenschädigung „bezahlt“ werden muss. Wie bei der Heisenberg'schen Unschärferelation in der Quantenphysik beeinflusst auch hier die Messung die zu messende Größe. Diese „Kosten“ durch das höhere Tidalvolumen in Form

von erhöhter Belastung der Lunge müssen gegen den zusätzlichen Informationsgewinn abgewogen werden. Bei einem $500 \mathrm{~g}$-Frühgeborenen mit einem optimalen Tidalvolumen von $2,5-3 \mathrm{ml}$ wird sich auch ein zusätzlicher Totraum von nur $1 \mathrm{ml}$ relevant auf die $\mathrm{CO}_{2}$-Elimination auswirken. Die Hersteller versuchen daher, die Toträume ihrer Sensoren möglichst gering zu halten. Kleinere Sensoren sind jedoch anfälliger für Sekret und Kondensat, was die Miniaturisierung wiederum begrenzt.

Diese Methoden umfassen:

- Messung der Thoraximpedanz

- Messung der Ausdehnung von Thorax oder Abdomen mit RIP-Bändern

- Feststellung der Abdominalbewegung über die sog. Graseby-Kapsel

- Ableitung von Aktionspotenzialen der Zwerchfellmuskulatur über eine im Ösophagus liegende, mit Elektroden besetzte Sonde (NAVA)

Eine weitere Möglichkeit für ein Triggersignal ist der durch die Inspiration des Patienten ausgelöste geringfügige Druckabfall im Beatmungsschlauchsystem (sog. Drucktrigger). 


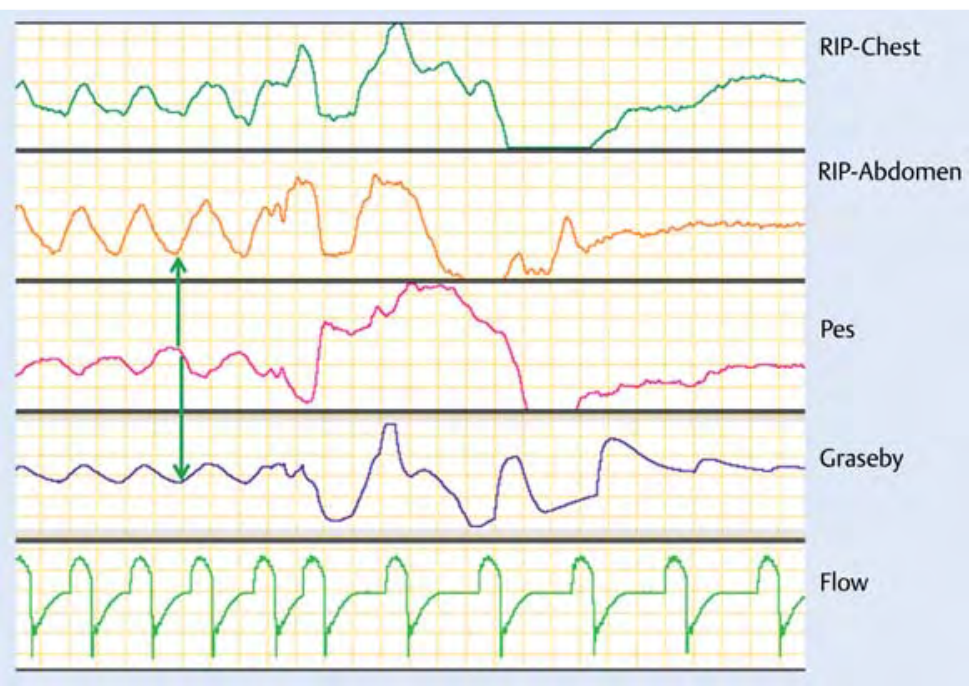

Abb.3 Vergleich der Signalverläufe verschiedener Atemsensoren. Man erkennt das sehr frühe Ansprechen der Signale von Graseby und RIP auf den Beginn einer Inspiration (erkennbar am Druckabfall im Ösophagus; die Pfleile markieren den Beginn einer Inspiration) im Vergleich zum Flowsignal (mit freundlicher Genehmigung von Prof. A. W. Flemmer, München).

\section{Lungenprotektive Beatmung}

Wie eine optimale lungenprotektive Beatmung beim Frühgeborenen aussieht, wurde bisher noch nicht in randomisierten Studien ermittelt. Zahlreiche Tierversuche von diversen Arbeitsgruppen konnten jedoch zeigen, dass die beatmungsbedingte Lungenschädigung wesentlich von der Größe des Tidalvolumens abhängt, nicht jedoch vom Beatmungsdruck, wenn der Druck nicht zu einem großen Tidalvolumen führt (Abb.4).

Drei vor der Einführung der Surfactantersatztherapie durchgeführte randomisierte Studien ergaben, dass bei gleichen Blutgaszielen in beiden Gruppen eine Beatmung mit einer Frequenz von 60 Atemzügen pro Minute weniger Pneumothoraces und interstitielle Emphyseme hervorruft als eine Beatmung mit 20-40 Atemzügen pro Minute. Da die Blutgasziele in beiden Gruppen gleich waren, ist davon auszugehen, dass die Frühgeborenen mit der höheren Beatmungsfrequenz und geringeren Komplikationsraten auch kleinere Tidalvolumina hatten, wobei nicht klar ist, wie groß die Tidalvolumina tatsächlich waren. Man kann diese Studien daher als Indiz für den Nutzen kleinerer Tidalvolumina interpretieren.

Merke: Bei kleinen Tidalvolumina, insbesondere bei einem Körpergewicht unter $1000 \mathrm{~g}$, gewinnt der Totraum eine erhebliche Relevanz, und jede technisch bedingte Totraumvergrößerung muss einer scharfen Risiko-Nutzen-Analyse unterzogen werden.

- Die Triggerung mithilfe der Graseby-Kapsel oder RIPBändern hat den Vorteil einer geringen zeitlichen Verzögerung, ist jedoch auch anfällig für Bewegungsartefakte, und die Platzierung des Sensors an der optimalen Stelle ist kritisch.

- NAVA erfordert das Einbringen einer relativ dicken und teuren Magensonde, was wiederum den Einsatz bei sehr kleinen Kindern ausschließt.

Nur eine der 3 verfügbaren großen Studien zur getriggerten Beatmung hat einen Vorteil gezeigt, bei allerdings sehr hoher basaler BPD-Rate (BPD: bronchopulmonale Dysplasie) [4]; dabei wurde die Graseby-Kapsel zur Triggerung eingesetzt. Die beiden anderen Studien ergaben gleiche Ergebnisse mit und ohne Synchronisierung $[5,6]$.

Merke: Der Nachweis, dass moderne Sensorik und moderne Beatmungsverfahren die Ergebnisse verbessern, steht noch aus.
Abb. 4 Schematische Darstellung, durch welche Volumenverläufe ein Volumentrauma entstehen kann. $\mathbf{A}$ hohes $V_{T}$, niedriger PEEP; B normales $V_{T}$, hoher PEEP; $C$ normales $V_{T}$, niedriger PEEP; D optimal. 


\section{Ergebnisse aus der Erwachsenen-} forschung

Eine große randomisierte Studie an erwachsenen Intensivpatienten mit dem akuten Atemnotsyndrom zeigte wesentlich günstigere Behandlungsergebnisse in der Gruppe mit einem Tidalvolumen von $6 \mathrm{ml} / \mathrm{kg}$, verglichen mit der Gruppe mit einem Atemzugvolumen von $12 \mathrm{ml} / \mathrm{kg}$ Körpergewicht. Interessanterweise hatte die Gruppe mit dem kleinen Tidalvolumen auch seltener mit dem Versagen anderer Organe zu kämpfen, was darauf hindeutet, dass eine durch Beatmung geschädigte Lunge vermutlich über die Freisetzung von Zytokinen erhebliche negative Fernwirkungen auf den gesamten Körper entfalten kann. Man geht heute davon aus, dass dies ebenso für lungenkranke Frühgeborene gilt, und dass ein Tidalvolumen von 4-6 ml/kg Körpergewicht auch für Frühgeborene und kranke Neugeborene den optimalen Bereich darstellt.

\section{Optimale Beatmungs- einstellungen finden}

\section{Inspiratorischer Spitzendruck}

Der inspiratorische Spitzendruck (PIP) sollte so eingestellt werden, dass der Thorax sich sichtbar, aber nur leicht hebt, und ein Tidalvolumen von 4-6 ml/kg Körpergewicht resultiert. Bei der Entwöhnung sollte immer erst der PIP und erst in zweiter Linie die Frequenz gesenkt werden.

\section{Beatmungsfrequenz}

Aufgrund der engen Grenzen für das Tidalvolumen kann ein adäquates Atemminutenvolumen i.d. R. nur mit relativ hohen Beatmungsfrequenzen erreicht werden. Der Steigerung der Beatmungsfrequenz sind jedoch durch die Physik ebenfalls Grenzen gesetzt, da ein Unterschreiten der minimal notwendigen Inspirationsund Exspirationszeiten zu einer unvollständigen Inspiration oder Exspiration führt. Während eine unvollständige Inspiration lediglich zu einem niedrigeren Tidalvolumen führt, kann es bei einer zu kurzen Exspiration zu Air Trapping in der Lunge und somit zu einer schleichenden Überblähung mit nachfolgendem Volutrauma kommen. Es ist also unbedingt darauf zu achten, dass die Exspirationszeit ausreichend lang ist.
Merke: Die Exspiationszeit sollte immer lang genug sein für eine vollständige Exspiration, um Air Trapping und nachfolgend Lungenschäden durch Überblähung zu vermeiden.

Ist die Exspirationszeit zu lang, geht Zeit verloren, und es werden weniger Atemzüge appliziert als möglich wären. Da das Atemzeitvolumen dennoch erreicht werden muss, werden die Atemzüge unter diesen Umständen größer ausfallen als nötig. Um mit möglichst kleinen Tidalvolumina auszukommen, sollte also die Exspirationszeit nicht unnötig lang sein.

Optimal ist also eine hohe Beatmungsfrequenz, mit noch ausreichender Exspirationszeit, kombiniert mit dem niedrigst möglichen PIP zur Erreichung der Blutgasziele.

Beil langer Exspirationszeit bleibt genug Zeit für eigene Atemzüge des Kindes. Reicht die Zeit allerdings nicht, um den selbst initiierten Atemzug abzuschließen, kommt es zur „Kollision“: Das Kind atmet noch aus, während das Gerät schon die nächste Einatmung beginnt. Beobachtet man dies, sollte die Exspirationszeit entweder verkürzt werden, um eine bessere Synchronisierung zu erreichen, oder so verlängert werden, dass zwischen 2 maschinellen Atemzügen genügend Zeit für einen eingefügten kindlichen Atemzug verbleibt.

Eine lange Inspirationszeit kann die Oxygenierung verbessern, aber auch eine Belastung für die Lunge darstellen. Bei kleinen Frühgeborenen mit Atemnotsyndrom kommt es nach meiner Erfahrung mit langen Inspirationszeiten häufiger zu einem pulmonalen interstitiellen Emphysem. Die Inspirationszeit sollte bei Frühgeborenen $<1500 \mathrm{~g}$ bei $0,3 \mathrm{~s}$, bei Frühgeborenen $<1000$ g standardmäßig bei 0,25s liegen. Alternativ könnte eine günstige Einstellung für die Inspirationszeit durch die Division des Gestationsalters durch 100 ermittelt werden.

Die Füllung der Lunge während der Inspiration und die Entleerung während der Exspiration folgen jeweils Exponentialfunktionen. Eine große Bedeutung für den genauen Verlauf dieser Exponentialfunktion hat die Zeitkonstante $\tau$, die sich aus der Multiplikation von Resistance und Compliance ergibt. Eine Zeitkonstante entspricht der Zeit, die für 63\% einer Inspiration oder Exspiration benötigt wird. Nach 3 Zeitkonstanten ist die Inspiration oder die Exspiration zu 95\%, nach 5 Zeitkonstanten zu 99\% abgeschlossen (Abb.5 und 6). 
Abb. 5 Berechnung und Wirkung der Lungenzeitkonstante.

Abb. 6 Auswirkung der Resistance auf den Exspirationsverlauf und die Exspirationszeit.
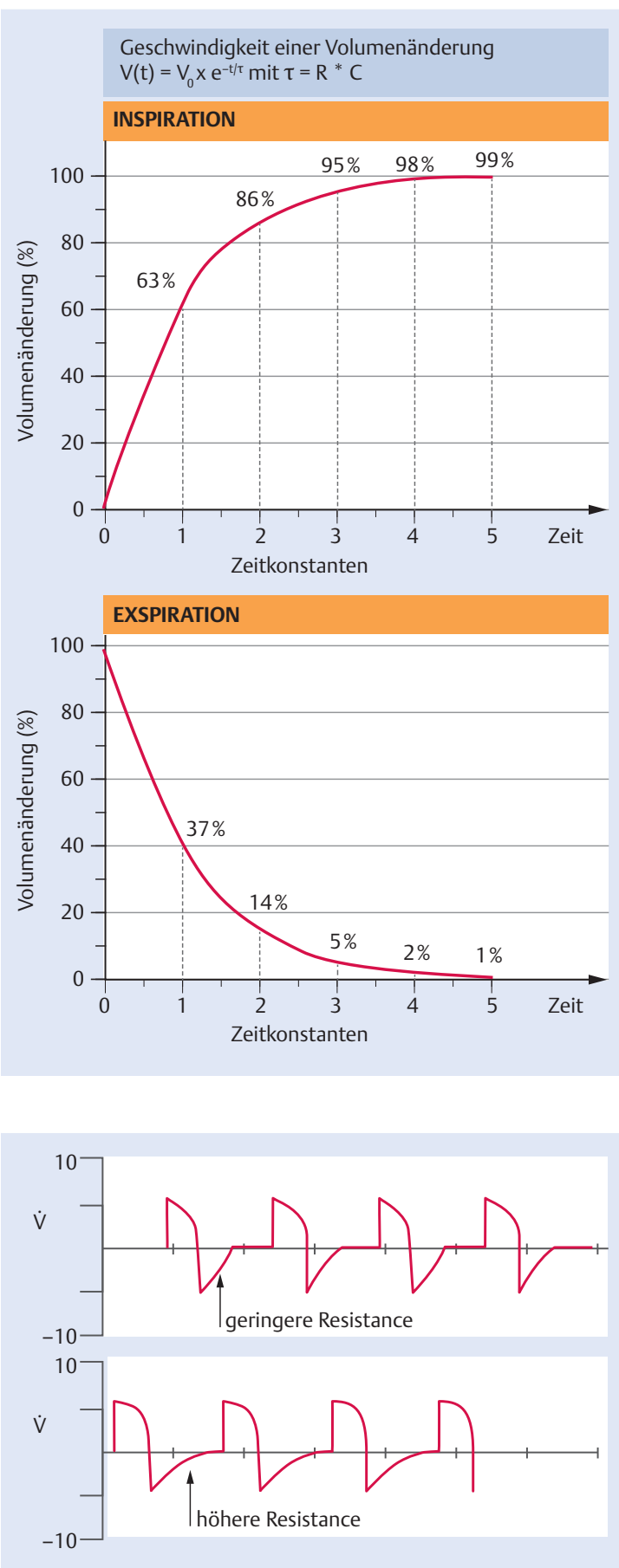

Merke: Ist bei hoher Resistance und/oder hoher Compliance der Lunge die Zeitkonstante entsprechend lang, wird auch für Inspiration und Exspiration eine entsprechend lange Zeit benötigt.

Frühgeborene mit Atemnotsyndrom weisen dagegen eine geringe Resistance und eine sehr geringe Compliance auf, was sehr kurze Zeitkonstanten ergibt, sofern der Tubus nicht sehr eng gewählt oder durch Sekrete verengt ist. Dies ermöglicht die Verwendung relativ kurzer In- und Exspirationszeiten und somit hohe Beatmungsfrequenzen bei der Beatmung Frühgeborener, und somit ergibt sich die Möglichkeit, durch hohe Frequenzen mit niedrigen Tidalvolumina auszukommen. Eine ausreichend lange Exspirationszeit für eine möglichst vollständige Exspiration bleibt jedoch essenziell, um eine Überblähung der Lunge durch Air Trapping zu vermeiden. Da der Strömungswiderstand inspiratorisch immer etwas geringer als exspiratorisch ist, sollte die Exspirationszeit immer etwas länger als die Inspirationszeit sein.

Merke: Die Zeitkonstante entspricht dem mathematischen Produkt von Compliance und Resistance. 3-5 Zeitkonstanten sind für jede Inspiration und Exspiration mindestens notwendig.

\section{Positiver endexspiratorischer Druck}

Zur Vermeidung des exspiratorischen Alveolarkollapses, insbesondere bei Surfactantmangel, spielt der positive endexspiratorische Druck (PEEP) nach wie vor die entscheidende Rolle. Er muss dafür ausreichend hoch eingestellt sein. Ein zu hoch eingestellter PEEP mit zu hohem endexspiratorischem Lungenvolumen kann jedoch, da in der Inspiration noch das Tidalvolumen zum endexspiratorischen Lungenvolumen hinzukommt, zu einer endinspiratorischen Lungenüberblähung und damit zum Volutrauma beitragen (Abb.7). Dies bedeutet, dass der PEEP individuell auf den einzelnen Patienten abgestimmt sein muss, und diese Abstimmung muss in regelmäßigen Abständen hinterfragt werden, da sich die mechanischen Eigenschaften der Lunge im Krankheitsverlauf verändern können. Randomisierte Studien verschiedener PEEP-Niveaus brachten kein eindeutiges Ergebnis, was jedoch nur unterstreicht, dass die Einstellung des PEEPs sehr individuell erfolgen muss, was sich sehr schlecht durch eine randomisierte Studie mit 2 Standardeinstellungen nachvollziehen lässt. 
Merke: Der PEEP muss für jeden Patienten individuell optimiert werden.

\section{Mittlerer Atemwegsdruck}

Die Oxygenierung des Patienten hängt v.a. vom mittleren Atemwegsdruck ab. Dieser wird durch verschiedene Beatmungsparameter unterschiedlich beeinflusst. Manche Parameter beeinflussen den mittleren Atemwegsdruck und das Atemminutenvolumen gleichsinnig, andere gegenläufig (Abb.8).

Merke: Der mittlere Atemwegsdruck bestimmt neben dem $\mathrm{FiO}_{2}$ die Oxygenierung.

\section{Zielwerte für Blutgase}

Unter Hyperventilation fällt der Partialdruck des $\mathrm{CO}_{2}$ $\left(\mathrm{pCO}_{2}\right)$ unter den Normbereich. Dies ist aus mehreren Gründen ungünstig und daher unbedingt zu vermeiden. Die Hyperventilation führt zu einer vermehrten Belastung des Lungengewebes und ist eindeutig mit der Entwicklung einer bronchopulmonalen Dysplasie assoziiert. Außerdem gibt es auch eine eindeutige Assoziation von abnorm niedrigen $\mathrm{pCO}_{2}$-Werten mit infantilen Zerebralparesen [7], was aus dem zerebral vasokonstriktorischen Effekt niedriger $\mathrm{pCO}_{2}$-Werte resultiert, die so eine zerebrale Ischämie auslösen. Der $\mathrm{pCO}_{2}$ sollte daher mind. im allgemein anerkannten Normalbereich liegen, günstigerweise sogar etwas darüber, um einen Sicherheitsabstand zur Hyperventilation einzuhalten. Noch höhere Ziele für den $\mathrm{pCO}_{2}$ könnten helfen, zusätzlichen Beatmungsdruck einzusparen und Volutrauma zu verhindern. Tierversuche und Untersuchungen an isolierten Lungenpräparaten ergaben zahlreiche Hinweise, dass ein erhöhter $\mathrm{pCO}_{2}$ mit respiratorischer Azidose protektiv für das Lungengewebe wirken kann. Außerdem kommt man durch Zulassen von höheren $\mathrm{pCO}_{2}$-Werten mit niedrigeren Tidalvolumina aus. Man nennt das permissive Hyperkapnie. Randomisierte Studien ergaben, dass sich leicht erhöhte Ziele für den $\mathrm{pCO}_{2}$ tatsächlich günstig auf die spätere Lungenfunktion auswirken können. Noch höhere Ziele für den $\mathrm{pCO}_{2}$ scheinen jedoch einer neueren Studie zufolge überwiegend nachteilig zu wirken [8]. Die Gründe hierfür sind noch unklar, vermutlich beeinträchtigt eine zu starke hyperkapnische Azidose doch wichtige Zellfunktionen zu sehr, wie z.B. die Reparatur von Epitheldefekten. Eine Kompensation niedriger $\mathrm{pH}$-Werte bei Hyperkapnie durch Puffergaben kommt nicht infrage, da

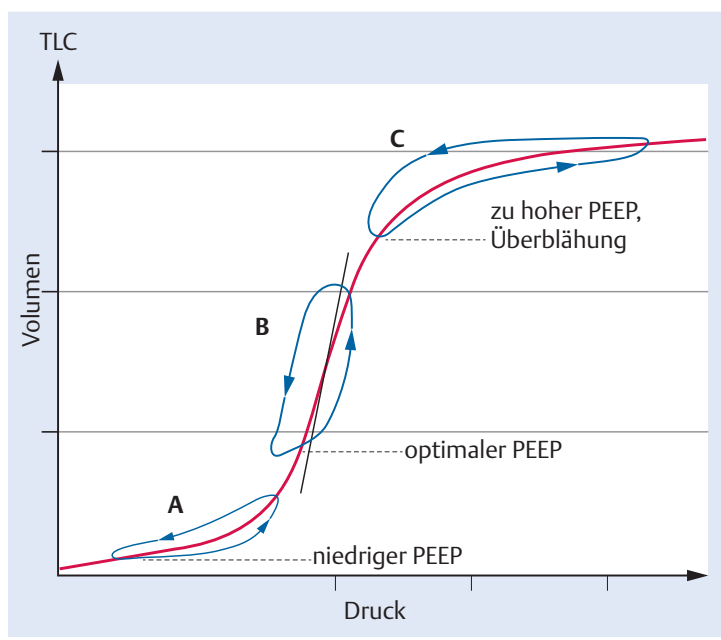

Abb. 7 Projektion verschiedener Druck-VolumenSchleifen auf die statische DruckVolumen-Kurve. A Zu niedriger PEEP, Lunge teilweise atelektatisch. B Optimal, größtmögliches Tidalvolumen mit geringstmöglicher Druckamplitude. C Zu hoher PEEP, Überblähung [1] (TLC: Total Lung Capacity).

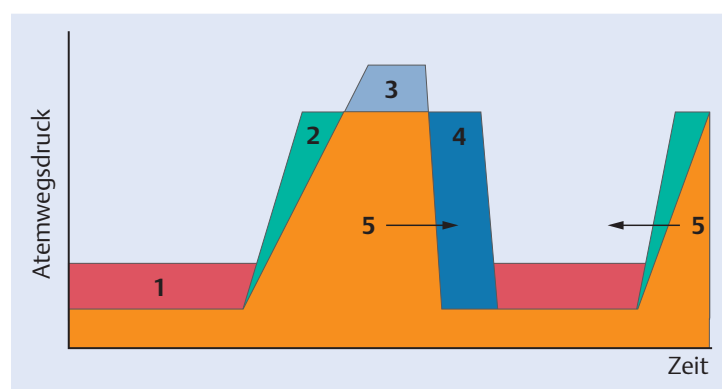

Abb. 8 Fünf Möglichkeiten, den mittleren Atemwegsdruck zu erhöhen: 1: höherer PEEP, 2: steilerer Druckanstieg, 3: höherer PIP, 4: längere Inspirationszeit, 5: kürzere Exspirationszeit $=$ höhere Frequenz.

Puffergaben sich in zahlreichen experimentellen Studien als lungenschädigend erwiesen haben [9]. Optimal scheint ein $\mathrm{pCO}_{2}$ in den ersten 3 Lebenstagen von 40-50 mmHg zu sein, der anschließend langsam weiter ansteigen darf, um den Atemantrieb zu erhöhen und die Entwöhnung von der Beatmung voranzutreiben.

Merke: Sehr hohe $\mathrm{pCO}_{2}$-Ziele bringen keine Vorteile gegenüber geringfügig erhöhten $\mathrm{pCO}_{2}$-Zielen.

\section{Patientengesteuerte Beatmungsmodi}

\section{Getriggerte Beatmungsmodi}

Patientengetriggerte Beatmungsmodi werden häufig auch als synchronisierte Modi bezeichnet. Bei der Betrachtung der synchronisierten Beatmungsmodi sollte nicht außer Acht gelassen werden, dass auch ohne Sensorik eine Synchronisierung erreicht werden kann. Das Einstellen einer maschinellen Beatmungsfrequenz, die in etwa der spontanen Atemfrequenz des Kindes entspricht, führt häufig dazu, dass 
sich das Kind mit der Beatmung selbst synchronisiert. Man spricht hierbei auch von Frequenzkopplung oder Entrainment. Beim Einsatz von Sensorik stehen verschiedene Modi zur Verfügung:

1. assistiert-kontrollierte Beatmung (A/C) bzw. synchronisierte intermittierende positive Druckbeatmung (SIPPV)

2. synchronisierte intermittierend-mandatorische Beatmung (SIMV)

3. druckunterstützte Beatmung (pressure support ventilation, PSV)

4. proportional assistierende Beatmung (PAV)

\section{- Assistiert-kontrollierte Beatmung (A/C) bzw. synchronisierte intermittierende positive Druckbeatmung (SIPPV)}

Dies bedeutet, dass bei jedem als Atembemühung interpretierten Signalverlauf des Sensors ein maschineller Atemhub mit den vorgewählten Einstellungen abgegeben wird. Dies ist eine sehr starke Form der Unterstützung, da der Patient jeweils nur den Trigger auslösen muss, um einen vollwertigen maschinellen Atemhub zu erhalten. Der Patient hat somit Kontrolle über seine Atemfrequenz, nicht aber über den Verlauf der einzelnen Atemhübe. Die Gefahr bei dieser Form der Unterstützung ist, dass Atemhübe auch durch Artefakte ausgelöst werden können, die das Triggersignal überlagern, sodass der Patient mehr Atemhübe bekommt als er eigentlich wollte. So kann es bei artefaktüberlagerten Signalen u.U. zu einer erheblichen Hyperventilation des Patienten kommen. Deshalb muss die korrekte Interaktion zwischen Kind und Beatmungsgerät regelmäßig überprüft und der $\mathrm{pCO}_{2}$ in geeigneter Weise überwacht werden.

Cave: Situationen, die zu einer Anreicherung des Triggersignals mit Artefakten führen (Wasser im Schlauchsystem), sollten zur Vermeidung einer gefährlichen Hyperventilation unbedingt vermieden werden.

\section{- Synchronisierte intermittierend- mandatorische Beatmung (SIMV)}

Bei dieser Beatmungsform erhält der Patient nur die voreingestellte Anzahl der Atemhübe mit maschineller Unterstützung. Das Beatmungsgerät versucht, diese Atemzüge möglichst dann abzugeben, wenn der Patient gerade auch eine spontane Einatmung ausführt. Dafür werden Zeitfenster definiert. Löst der Patient innerhalb des Zeitfensters den Trigger aus, erhält er einen mechanischen Atemhub. Löst er den Trigger nicht aus, erhält er am Ende des Zeitfensters ebenfalls einen mechanischen Atemhub, in diesem Falle ohne Synchronisierung zu seinen eigenen Atembemühungen. Löst der Patient den Trigger häufiger aus, als es die voreingestellte Atemfrequenz vorgibt, so werden die zusätzlichen Atemzüge nicht unterstützt, sondern müssen auf dem Niveau des endexspiratorischen Druckes vom Patienten selbst ausgeführt werden. Der Patient hat somit eine begrenzte Kontrolle über den Zeitpunkt der maschinell applizierten Atemhübe, aber nicht über die Frequenz, sondern er erhält nur die voreingestellte Anzahl. Dafür kann er zusätzliche Atemzüge ohne Zutun der Maschine komplett selbst steuern, bekommt aber hierfür keine Unterstützung. Die Gefahr einer durch Triggerartefakte ausgelösten Hyperventilation ist deutlich geringer, dafür ist das Maß der maschinellen Unterstützung aber auch reduziert, je nachdem wie hoch die maschinelle Beatmungsfrequenz eingestellt ist.

\section{- Druckunterstützte Beatmung (PSV)}

Diese Beatmungsform erfordert einen Flowsensor, mit anderen Triggersystemen ist sie nicht durchführbar. Auch hier wird die maschinelle Inspiration des Patienten synchronisiert mit einer Spontaninspiration begonnen und das Druckanstiegsprofil und der inspiratorische Spitzendruck sind durch die Einstellung des Beatmungsgeräts festgelegt. Für die Inspirationsdauer gibt es jedoch nur einen Maximalwert, die tatsächliche Inspirationsdauer endet, wenn der Inspirationsfluss einen bestimmten Grenzwert unterschreitet. Das bedeutet, sobald die Luft nur noch sehr langsam in die Lunge hineinströmt, wird die Inspiration abgebrochen und der Druck auf das endexspiratorische Niveau abgesenkt. Der Patient hat dadurch Einfluss auf die Inspirationsdauer. Er muss nicht mehr, wenn er seine Inspiration schneller beenden möchte, als es die eingestellte Inspirationsdauer vorsieht, gegen den Inspirationsdruck ausatmen. Druckunterstützte Beatmung kann auch mit den vorher genannten Modi kombiniert werden. Kombiniert mit assistierend-kontrollierter Beatmung werden alle Atemzüge nach dem Prinzip des „Pressure Supports“ gesteuert und haben eine variable Inspirationsdauer. Mit SIMV kombiniert ist es möglich, eine bestimmte Anzahl von Atemzügen mit fester Inspirationszeit vorzugeben und die vom Patienten zusätzlich durchgeführten Atemzüge nach dem PSVPrinzip zu unterstützen. Je nach Beatmungsgerät kann für die beiden Arten von Atemzügen mitunter auch ein unterschiedlicher Inspirationsdruck vorgewählt werden. 


\section{Proportional assistierende Beatmung (PAV)}

Bei diesem Modus wird die Eigenatmung des Patienten kontinuierlich proportional unterstützt. Proportional bedeutet eine Regulation des Atemwegdrucks entweder proportional zum inspiratorischen Fluss, was dem Patienten im Vergleich zur reinen Spontanatmung die Überwindung der Atemwegsresistance erleichtert, oder proportional zum inspirierten Volumen, was für den Patienten den Kraftaufwand zur Dehnung der Lunge verringert (Abb.9 und 10). Beides kann auch kombiniert werden. Der Patient atmet völlig frei, und er wird in jeder Phase proportional unterstützt. Somit hat er die volle Kontrolle über Atemfrequenz, Atemzugvolumen und den zeitlichen Verlauf eines jeden Atemzugs. Das Ausmaß der Verstärkung der Atembemühungen des Patienten muss jedoch korrekt eingestellt werden, da die Regulation des Atemwegsdrucks durch die Maschine sonst instabil werden kann. Ein weiterer Nachteil ist, dass der Patient nur dann unterstützt wird, wenn er auch selbst Atembemühungen hat. Im Falle einer Apnoe wird auch keine maschinelle Unterstützung gegeben, was etwa vergleichbar mit einem Pedelec ist. Nur wenn der Fahrer in die Pedale tritt, erfolgt eine Unterstützung durch den elektrischen Antrieb.

Merke: Für die PAV bedeutet dies, dass zur Überbrückung von Apnoen unbedingt eine im Beatmungsgerät einstellbare Back-up-Funktion vorhanden sein muss, die den Patienten in den Phasen, in denen er nicht selbst atmet, kontrolliert beatmet.

\section{nutzen und Risiken der getriggerten Modi}

Über den Nutzen synchronisierter Beatmungsverfahren gab es 3 große randomisierte Studien. Nur eine Studie mit totraumfreier Triggerung durch eine Graseby-Kapsel fand eine Verringerung der bronchopulmonalen Dysplasie im Zusammenhang mit der synchronisierten Beatmung, allerdings bei einer insgesamt sehr hohen BPD-Rate, was die Übertragbarkeit der Ergebnisse auf heutige Verhältnisse einschränkt [4]. In den beiden anderen Studien waren die Ergebnisse bei synchronisierter und unsynchronisierter Beatmung gleich $[5,6]$, sodass diese Frage nicht geklärt ist. Der Nutzen von PSV und PAV wurde noch gar nicht in einer großen randomisierten Studie untersucht. Kleinere Studien berichten von einem geringeren Bedarf an Beatmungsdruck; ob dies allerdings tatsächlich zu besseren Behandlungsergebnissen führt, ist derzeit noch völlig unklar. Es ist daher noch offen, ob patientengesteuerte Modi zu besseren Ergebnissen führen. Eine umgekehrte Argumentation, dass es fehlerhaft ist, auf patientengesteuerte Beatmung zu verzichten, ist ebenso nicht möglich. Entscheidend ist auch in dieser Frage die

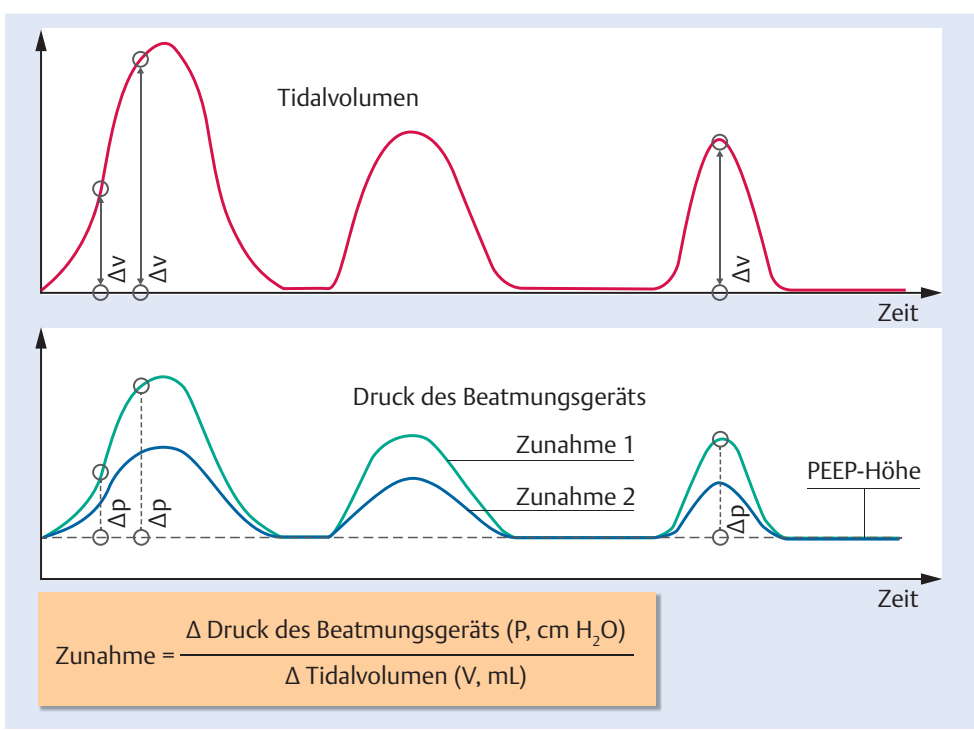

Abb.9 Volumen-proportional-assistierende Beatmung: entlastet den Patienten bei der Überwindung der elastischen Rückstellkräfte seiner Lunge [10].

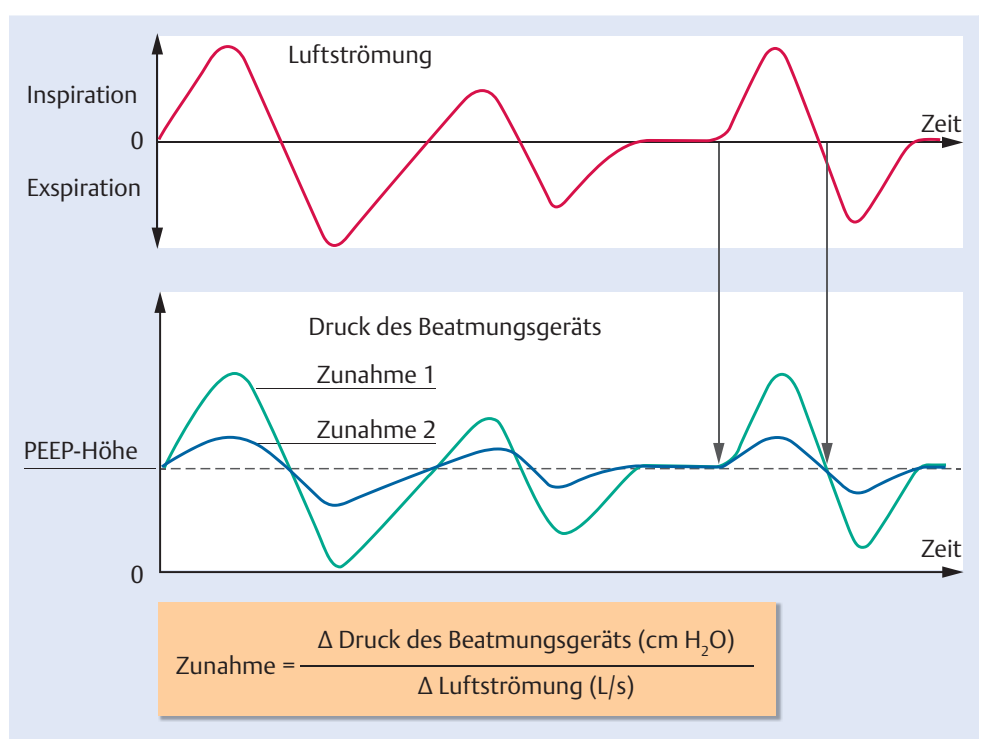

Abb.10 Flow-proportional-assistierende Beatmung: entlastet den Patienten bei der Überwindung des Atemwegswiderstands [10].

jeweilige Abwägung von Risiken und Nutzen. Die Risiken beziehen sich in diesem Falle auf die durch manche Triggersysteme erzeugte Totraumerhöhung, die ja durch die Beatmung kompensiert werden muss. Der Nutzen ist eventuell erkennbar anhand der Apnoehäufigkeit oder anhand des subjektiven Eindrucks über den Komfort des Patienten. 


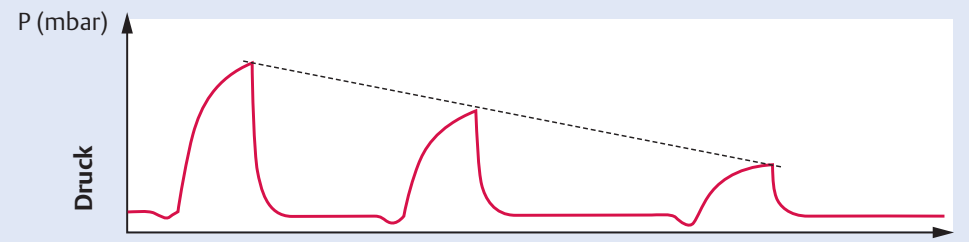

Zeit (s)
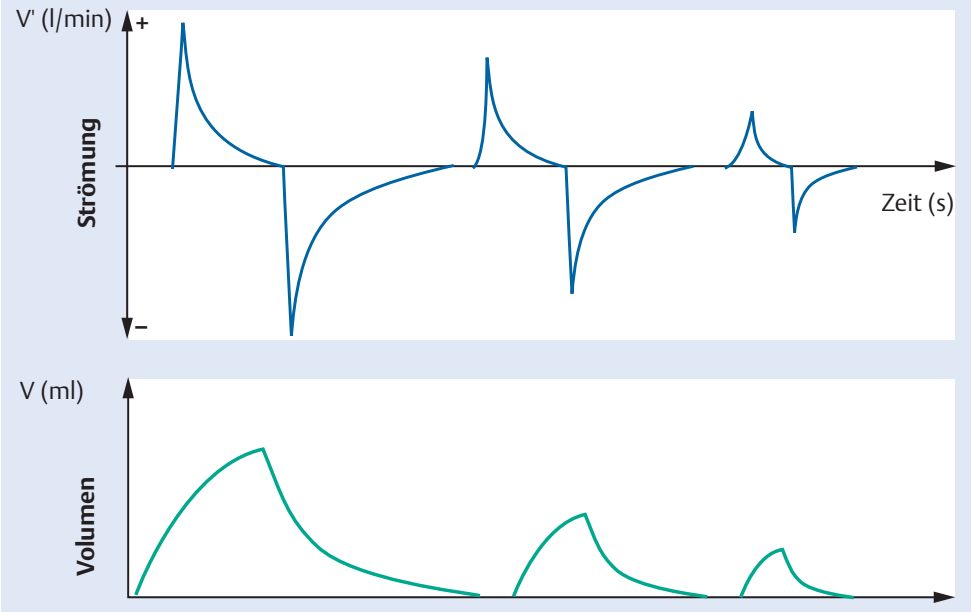

Abb.11 Funktionsweise der Volumengarantie-Beatmung. Liegt das ausgeatmete Tidalvolumen über dem Sollwert, wird der Beatmungsdruck automatisch schrittweise abgesenkt. Fällt das exspiratorische Tidalvolumen unter den Sollwert, wird der Druck wieder angehoben. die Größe des von der Maschine gemessenen inspirierten Tidalvolumens und des tatsächlich in die Lunge gelangten Atemzugvolumens stark voneinander abweichen können.

\section{- Volumengarantierte Beatmung}

Bei der volumengarantierten Beatmung erfolgt die Steuerung nach dem exspiratorischen Tidalvolumen, was wesentlich besser mit dem tatsächlichen Atemzugvolumen des Patienten korreliert. Dies liegt daran, dass die Exspiration bei niedrigerem Atemwegsdruck erfolgt und damit die Volumenverluste durch ein etwaiges Tubusleck deutlich geringer ausfallen. Das Problem hierbei ist, dass das exspiratorische Volumen erst bekannt ist, wenn der Atemzug schon vorüber ist. Der Messwert kann sich daher nur auf die nachfolgenden Atemzüge auswirken. Das Beatmungsgerät steuert also den Inspirationsdruck der nachfolgenden Atemzüge anhand des Vergleichs zwischen dem tatsächlich exspirierten Tidalvolumen und dem vom Benutzer eingestellten Sollwert. Die einzelnen Atemzüge erfolgen drucklimitiert, da die Maschine den Inspirationsdruck vorher festlegt und dann den Atemhub entsprechend ausführt.

Damit ist die Volumengarantie im Prinzip eine drucklimitierte Beatmung mit Steuerung anhand eines Volumenziels. Meistens erfolgt die Anpassung durch gleitende Mittelwertbildung über mehrere Atemzüge. Bei diesem Verfahren wird das Beatmungsgerät in Phasen guter Mitarbeit des Patienten den Beatmungsdruck schrittweise automatisch absenken, während in Phasen, in denen der Patient schlecht mitatmet oder gegen die Beatmung „presst“, mit einem höheren Beatmungsdruck ein angestrebtes Tidalvolumen in etwa aufrechterhalten werden kann (Abb.11).

Mehrere kleinere Studien ergaben, dass die Häufigkeit von Phasen mit niedriger Sauerstoffsättigung bei beatmeten Patienten unter Volumengarantie geringer ist und außerdem die Beatmung im Schnitt mit einem geringeren Beatmungsdruck auskam, als dies bei einer gleich effektiven druckkontrollierten Beatmung der Fall war $[11,12]$. Es kann spekuliert werden, dass es durch die unablässig von der Maschine durchgeführten Versuche, den Beatmungsdruck zu reduzieren, außerdem zu einer schnelleren Entwöhnung des Patienten von der Beatmung kommt. Weiterhin kann spekuliert werden, dass dieses Verfahren aufgrund der ständig von der Maschine angestrebten niedrigstmöglichen Beatmungsdrucke für die Patientenlunge sehr schonend ist, trotz des zusätzlichen, für den Flowsensor erforder- 
lichen Totraums. Große randomisierte Studien fehlen jedoch auch hierzu.

Merke: Volumengarantie ist eine drucklimitierte Beatmung, bei der der Inspirationsdruck automatisch anhand eines Vergleichs der vorangegangenen exspiratorischen Tidalvolumina mit einem Tidalvolumensollwert nachgeregelt wird.

\section{Nichtinvasive Beatmung}

Unter nichtinvasiver Beatmung werden alle Formen der Atemunterstützung mithilfe von Überdruck zusammengefasst, die ohne einen endotracheal platzierten Tubus auskommen. Es sind verschiedene Varianten möglich, diese unterscheiden sich in der:

- Druckverlaufskurve

- Art der Druckerzeugung

- Methode, wie der Überdruck auf den Patienten übertragen wird (,interface“)

\section{Druckverlauf}

Möglich sind ein gleichmäßiger Druck (continuous positive airway pressure, CPAP) oder ein wechselndes Druckniveau mit höheren Druckphasen für die Inspiration und niedrigeren Druckphasen für die Exspiration. Diese Form wird überwiegend mit NIPPV abgekürzt (nichtinvasive positive Druckbeatmung). Steht ein Triggersignal zur Verfügung, kann NIPPV auch synchronisiert mit den patienteneigenen Atemanstrengungen als SNIPPV-Therapie appliziert werden. Ein großes Leck erschwert eine Triggerung über das Beatmungssystem, für diesen Fall stehen andere Triggersysteme zur Verfügung, z.B. die Graseby-Kapsel. Studien zufolge ist dies die wirksamste Art der nichtinvasiven Unterstützung [13].

In Studien wurde gezeigt, dass eine nichtinvasive Beatmung wirksamer ist als reiner CPAP [16]. Klinisch relevante Unterschiede zwischen den verschiedenen CPAP-Arten konnten bisher nicht nachgewiesen werden. Wichtig ist in jedem Fall eine ausreichende Anfeuchtung.

\section{Art der Druckerzeugung}

Die einfachste Möglichkeit der nichtinvasiven Beatmung besteht darin, über eine sog. Nasenbrille einen kontinuierlichen Luftstrom in die Nasenlöcher zu applizieren. Man bezeichnet dies als Hochflussnasenkanüle (heated humidified high flow nasal cannula, HHHFNC) oder „Mini-CPAP“. Dabei entsteht im NasenRachen-Raum ein Überdruck, dessen Höhe allerdings bei dieser einfachen Methode nicht gemessen werden kann.

Merke: Für den Patienten ist dies die angenehmste Beatmungsform, da sie mit der geringsten apparativen Beeinträchtigung der Gesichtsregion einhergeht $[14,15]$.

Eine weitere Methode der Druckerzeugung ist das sog. Infant-Flow-System. Hierbei wird in einem sog. Generator, der auf die Nase aufgesetzt wird, ein dünner, kräftiger Gasfluss in den Nasenaufsatz eingebracht. Dabei entsteht auf dynamischem Weg ein erhöhter Druck, der sich auf den Nasen-Rachen-Raum überträgt und der auch gemessen werden kann. Da diese Methode der Druckerzeugung ohne Ventile auskommt, ist der Atemwiderstand sehr gering, was für das betroffene Kind eine optimal geringe Atemarbeit bedeutet. Durch die zyklische Variation des Gasstroms kann auch eine zyklische Druckänderung hervorgerufen werden, sodass eine nichtinvasive Beatmung und bei Synchronisation mit der Graseby-Kapsel sogar eine synchronisierte nichtinvasive Beatmung möglich ist.

Merke: Leider sind die erreichbaren Druckamplituden nicht sehr groß, sodass die Effektivität dieser SNIPPV-Form geringer ist als die mit echten Beatmungsgeräten.

Ein weiterer Vorteil sind die geringen Kosten eines Infant-Flow-Geräts im Vergleich zu einem echten Beatmungsgerät. Ein Nachteil des Systems ist die durch die starke Verwirbelung entstehende Geräuschentwicklung, sodass ein Infant-Flow-CPAP deutlich lauter ist als die anderen hier diskutierten Methoden.

Die 3. Möglichkeit besteht in einem Schlauchsystem mit konstantem Fluss vorgewärmter Luft oder eines Luft-Sauerstoff-Gemischs, das in einem Wasserschloss endet, der sog. Bubble CPAP. Die Wassertiefe bestimmt den CPAP-Druck. Das unablässige „Blubbern“ erzeugt hochfrequente Schwingungen mit geringer Amplitude. Ob diese helfen, den Gasaustausch zu verbessern, konnte bisher nicht zweifelsfrei nachgewiesen werden. 
Merke: Wichtig ist, dass so viel Flow angeboten wird, dass das Kind auch bei kräftiger Inspiration diesen Flow nicht zu überschreiten vermag, denn sonst würde es das Schlauchsystem „leer saugen“ und der CPAP-Druck würde zusammenbrechen.

Für das Kind wäre das vermutlich mit dem Gefühl von Atemnot verbunden. Das System ist sehr preiswert, jedoch ist die Einstellung rasch wechselnder Druckniveaus, wie bei einer Beatmung, nicht möglich.

Die 4. Möglichkeit einer nichtinvasiven Beatmung besteht im Einsatz eines echten Beatmungsgeräts. Wesentliche Voraussetzung ist, dass das Beatmungsgerät hinreichende Flussraten zur Verfügung stellen kann, um das bei nichtinvasiver Beatmung bestehende große Leck zu kompensieren. Mit dem Beatmungsgerät kann ein kontinuierlicher Atemwegsdruck (CPAP) oder auch eine nichtinvasive Beatmung appliziert werden. Falls das Gerät über Triggermöglichkeiten verfügt, die ohne einen Flowsensor auskommen, kann auch eine synchronisierte nichtinvasive Beatmung durchgeführt werden. Eine Triggerung mit dem Flowsensor ist aufgrund des großen Leckes i.d.R. nicht mit ausreichender Zuverlässigkeit möglich, wenngleich mehrere Firmen versuchen, diese Probleme mithilfe intelligenter Algorithmen in den Griff zu bekommen. Das Beatmungsgerät bietet somit die umfangreichsten Möglichkeiten der nichtinvasiven Unterstützung, allerdings ist dies aufgrund der hohen Gerätekosten auch die teuerste Methode. Je nach Schnelligkeit der im Gerät eingebauten Ventilmechanismen kann es sein, dass die Atemarbeit für den Patienten etwas höher ist als mit dem Infant-Flow-System.

\section{Möglichkeiten für das Patienten-Interface}

HHFNC wird nur über die Nasenbrille appliziert. Für das Infant-Flow-System und für die Beatmungsgeräte stehen wahlweise binasale Prongs oder Nasenmasken zur Verfügung. Studien zufolge funktionieren beide gleich gut, es kommt auf das jeweilige Kind an, welche Methode die geeignetere ist. Da beides Druckstellen an der Nase hervorrufen kann, ist es oftmals am günstigsten, die Methoden regelmäßig zu wechseln, damit sich die Haut an den jeweiligen Stellen wieder erholen kann. Wird ein Beatmungsgerät eingesetzt, kann die nichtinvasive Beatmung auch über einen im Nasopharynx liegenden Endotrachealtubus (sog. pharyngealer Tubus oder Rachentubus) appliziert werden. Da hierfür nur ein Nasenloch genutzt wird und außerdem der Tubus so klein sein muss, dass er durch die Nase passt, ist der Strömungswiderstand höher als bei den binasalen Prongs oder Nasenmasken. Dementsprechend haben mehrere Studien und Metaanalysen ergeben, dass die Atemunterstützung mit dem mononasalen Tubus weniger effektiv ist. Ein Vorteil dieser Methode ist die wesentlich einfachere Fixierung am Kind.

\section{Nutzen der nichtinvasiven Beatmung}

Der Nutzen von nichtinvasiver Beatmung wurde in zahlreichen Studien untersucht. Bei Vergleichen stellte sich heraus, dass die Applikation von nasalem CPAP unter Verzicht auf eine Surfactantgabe gleich gute Behandlungsergebnisse erzielte wie die primäre Intubation zur Surfactantgabe mit nachfolgender Beatmung $[17,18]$. Eine bei einem Teil der Studien beobachtete höhere Pneumothoraxrate mit CPAP führte nicht zu einem schlechteren Langzeitoutcome. Dies zeigt, dass nasaler CPAP eine effektive und schonende Methode der Atemunterstützung für Frühgeborene darstellt.

Eine weitere Optimierung stellt die in den letzten Jahren von Kribs und Göpel entwickelte Surfactantgabe ohne Intubation und mechanische Beatmung dar. Hierbei wird unter nasalem CPAP ein dünner Schlauch in die Trachea vorgeschoben und über diesen der Surfactant appliziert, den das Kind dann mit seiner Eigenatmung in die Lunge transportiert. In mehreren randomisierten Studien konnte gezeigt werden, dass diese Methode zu besseren Behandlungsergebnissen führt als reiner CPAP ohne Surfactant oder die Surfactantgabe unter Intubation und mechanischer Beatmung $[19,20]$.

Merke: Bei einem Neugeborenen mit gut ausgeprägter Eigenatmung stellt CPAP mit Surfactantgabe ohne Intubation wahrscheinlich die schonendste Form der Unterstützung dar.

Weitere Metaanalysen zeigten, dass die Vermeidung einer invasiven Beatmung, ganz gleich mit welcher Methode, zu besseren Behandlungsergebnissen führte als in den jeweiligen Kontrollgruppen [21]. Es kam zu einer Verbesserung der Überlebensrate ohne BPD und ohne Anstieg anderer Komplikationen, wie z.B. intraventrikuläre Blutungen.

Relativ neu ist die Idee, nichtinvasiven CPAP mit Hochfrequenzoszillation zu kombinieren. Erste Versuche zeigten, dass hochfrequente Oszillationen, die auf den nasalen CPAP aufgebracht werden, zu einer signifikan- 
ten Reduktion des Kohlendioxidpartialdrucks führen [22]. Die Oxygenierung bessert sich dagegen nicht.

Merke: Die nichtinvasive Beatmung kann oft die invasive Beatmung ersetzen und verringert dabei die Komplikationsrate.

\section{Zusammenfassung}

Bei der konventionellen Beatmung sollten kleine Tidalvolumina angestrebt werden, wobei hohe Beatmungsfrequenzen helfen. Die Synchronisierung der Beatmung kann den Patientenkomfort verbessern, der Nachweis eines besseren „Langzeitoutcomes“ steht jedoch noch aus. Die Kontrolle und Regelung des Tidalvolumens anstelle einer festen Druckbegrenzung könnte die Behandlungsergebnisse verbessern, diese Vorteile müssen jedoch gegen den durch einen Flowsensor verursachten höheren Totraum abgewogen werden. Das Vermeiden von invasiver Beatmung zugunsten von nichtinvasiver Beatmung ist mit besseren Ergebnissen assoziiert, hierfür wurden verschiedene Methoden entwickelt.

Interessenkonflikt: Kein Interessenkonflikt angegeben.

\section{Über den Autor}

\section{Ulrich H. Thome}

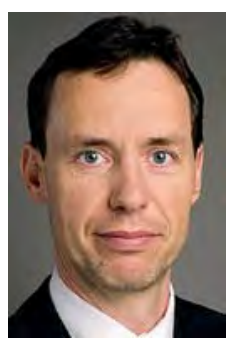

Jahrgang 1964, Univ.-Prof. Dr. med. Nach Medizinstudium und Promotion in Düsseldorf Ausbildung als Kinderarzt und pädiatrischer Intensivmediziner an der Universitätskinderklinik Ulm. Ab 1998 für 3 Jahre an der Universität von Alabama in Birmingham (USA) zur Forschung und gleichzeitig Ausbildung zum Neonatologen. Nach Rückkehr Oberarzt und Habilitation an der Universität Ulm. Seit 2008 Leiter der selbständigen Abteilung für Neonatologie am Universitätsklinikum Leipzig. Weiterbildungsermächtigung für Neonatologie und Pädiatrische Intensivmedizin.

\section{Korrespondenzadresse}

Prof. Dr. med. Ulrich H. Thome

Facharzt für Kinder- und Jugendmedizin/Neonatologie/

Pädiatrische Intensivmedizin

Abteilung Neonatologie der Universitätsklinik für

Kinder- und Jugendmedizin

Zentrum für Frauen- und Kindermedizin

Liebigstr. 20a, Haus 6

04103 Leipzig

ulrich.thome@medizin.uni-leipzig.de

\section{Literatur}

1 Harris TR et al. Physiologic Principles. In: Goldsmith JP, Karotkin EH. Assisted ventilation of the neonate. 3rd ed. Saunders/ Elsevier; 1996

2 The Acute Respiratory Distress Syndrome Network. Ventilation with lower tidal volumes as compared with traditional tidal volumes for acute lung injury and the acute respiratory distress syndrome. N Engl J Med 2000; 342: 1301 - 1308

3 Bolivar JM, Gerhardt T, Gonzalez A et al. Mechanisms for episodes of hypoxemia in preterm infants undergoing mechanical ventilation. J Pediatr 1995; 127: 767-773

4 Bernstein G, Mannino FL, Heldt GP et al. Randomized multicenter trial comparing synchronized and conventional intermittent mandatory ventilation in neonates. J Pediatr 1996; 128: $453-463$

5 Baumer JH. International randomised controlled trial of patient triggered ventilation in neonatal respiratory distress syndrome. Arch Dis Child Fetal Neonatal Ed 2000; 82: 5-10

6 Beresford MW, Shaw NJ, Manning D. Randomised controlled trial of patient triggered and conventional fast rate ventilation in neonatal respiratory distress syndrome. Arch Dis Child Fetal Neonatal Ed 2000; 82: 14-18

7 Laffey JG, Kavanagh BP. Hypocapnia. N Engl J Med 2002; 347 : $43-53$

8 Thome UH, Genzel-Boroviczeny O, Bohnhorst B et al. Permissive hypercapnia in extremely low birthweight infants (PHELBI): a randomised controlled multicentre trial. Lancet Respir Med 2015; 3: 534 - 543

9 Hummler HD, Engelmann A, Merinsky A et al. Buffering Acidosis During Permissive Hypercapnia To Restore Physiologic pH May Increase the Degree of Lung Injury in Surfactant Deficient Rabbits. Pediatr Acad Soc 2004; 55: 2886

10 Hummler $\mathrm{H}$, Schulze A. New and alternative modes of mechanical ventilation in neonates. Semin Fetal Neonatal Med 2009; 14: $42-48$

11 Duman N, Tuzun F, Sutcuoglu S et al. Impact of volume guarantee on synchronized ventilation in preterm infants: a randomized controlled trial. Intensive Care Med 2012; 38: 1358 1364

12 Erdemir A, Kahramaner Z, Turkoglu E et al. Effects of synchronized intermittent mandatory ventilation versus pressure support plus volume guarantee ventilation in the weaning phase of preterm infants. Pediatr Crit Care Med J Soc Crit Care 
Med World Fed Pediatr Intensive Crit Care Soc 2014; 15: 236 241

13 Gizzi C, Montecchia F, Panetta $V$ et al. Is synchronised NIPPV more effective than NIPPV and NCPAP in treating apnoea of prematurity (AOP)? A randomised cross-over trial Arch Dis Child Fetal Neonatal Ed 2015; 100: 17-23

14 Yoder BA, Stoddard RA, Li M et al. Heated, humidified highflow nasal cannula versus nasal CPAP for respiratory support in neonates. Pediatrics 2013; 131: 1482-1490

15 Manley BJ, Owen LS, Doyle LW et al. High-flow nasal cannulae in very preterm infants after extubation. N Engl J Med 2013; 369: $1425-1433$

16 Li W, Long C, Zhangxue $\mathrm{H}$ et al. Nasal intermittent positive pressure ventilation versus nasal continuous positive airway pressure for preterm infants with respiratory distress syndrome: a meta-analysis and up-date. Pediatr Pulmonol 2015; 50: $402-409$

17 Morley CJ, Davis PG, Doyle LW et al. Nasal CPAP or intubation at birth for very preterm infants. N Engl J Med 2008; 358 : $700-708$
18 Finer NN, Carlo WA, Walsh MC et al. Early CPAP versus surfactant in extremely preterm infants. N Engl J Med 2010; 362 : $1970-1979$

19 Gopel W, Kribs A, Ziegler A et al. Avoidance of mechanical ventilation by surfactant treatment of spontaneously breathing preterm infants (AMV): an open-label, randomised, controlled trial. Lancet 2011; 378: 1627-1634

20 Kribs A, Roll C, Göpel W et al. Nonintubated Surfactant Application vs Conventional Therapy in Extremely Preterm Infants: A Randomized Clinical Trial. JAMA Pediatr 2015; published online June 8; DOI: 10.1001/jamapediatrics.2015.0504

21 Fischer HS, Bührer C. Avoiding endotracheal ventilation to prevent bronchopulmonary dysplasia: a meta-analysis. Pediatrics 2013; 132: e1351 - e1360

22 Czernik C, Schmalisch G, Buhrer C et al. Weaning of neonates from mechanical ventilation by use of nasopharyngeal high-frequency oscillatory ventilation: a preliminary study. J Matern Fetal Neonatal Med 2012; 25: 374-378

\section{Erratum}

Dieser Artikel wurde gemäß dem Erratum vom 18.12.2015 geändert.

Auf Seite $339 \mathrm{im} \mathrm{3.} \mathrm{Absatz} \mathrm{heißt} \mathrm{es} \mathrm{jetzt} \mathrm{anstelle} \mathrm{von:}$ Optimal ist also eine hohe Beatmungsfrequenz, mit noch ausreichender Inspirationszeit, kombiniert mit dem niedrigst möglichen PIP zur Erreichung der Blutgasziele.

Optimal ist also eine hohe Beatmungsfrequenz, mit noch ausreichender Exspirationszeit, kombiniert mit dem niedrigst möglichen PIP zur Erreichung der Blutgasziele. 


\section{CME-Fragen}

\section{CME.thieme.de}

CME-Teilnahme

- Viel Erfolg bei Ihrer CME-Teilnahme unter http://cme.thieme.de

- Diese Fortbildungseinheit ist 12 Monate online für eine CME-Teilnahme verfügbar.

- Sollten Sie Fragen zur Online-Teilnahme haben, unter http://cme.thieme.de/hilfe finden Sie eine ausführliche Anleitung.

1

Eine schonende Beatmung hat folgende Eigenschaft:
A großes Tidalvolumen
B kleines Tidalvolumen
C hoher positiver endexspiratorischer Druck
D niedriger positiver endexspiratorischer Druck
E niedrige Beatmungsfrequenz

\section{2}

Welche Errungenschaft senkt erwiesenermaßen das Risiko für eine BPD?
A Flowsensor
B Triggerung
C Computersteuerung
D großes Display mit Atemkurven
E CPAP/nichtinvasive Beatmung

\section{3}

Welcher Einstellungsfehler verursacht keine vermehrten Schäden an der Lunge?
A zu hoher Inspirationsdruck
B zu hoher positiver endexspiratorischer Druck
C zu niedriger positiver endexspiratorischer Eindruck
D zu lange Inspirationszeit
E zu niedriger Inspirationsdruck

Welche Triggermethode ist anfällig für Lecks?
A Flowsensor
B Graseby-Kapsel
C Impedanzmessung
D RIP-Bänder
E NAVA

5

Welche Aussage ist falsch? Die nichtinvasive Beatmung
A ist assoziiert mit einem Rückgang der BPD-Rate.
B ist assoziiert mit einer höheren Pneumothoraxrate.
C kann mit getriggerten Beatmungsmodi kombiniert werden.
D war in Studien nicht schlechter als invasive Beatmung mit Surfactantgabe.
E erfordert keine Anfeuchtung der Atemluft.

6

Der Strömungswiderstand in den Atemwegen
A ist bei Inspiration und Exspiration gleich.
B ist bei Inspiration höher als bei Exspiration.
C ist bei Inspiration niedriger als bei Exspiration.
D ist bei Frühgeborenen mit Atemnotsyndrom besonders hoch.
E hat für die Wahl der Beatmungsparameter keine wesentliche Bedeutung. 


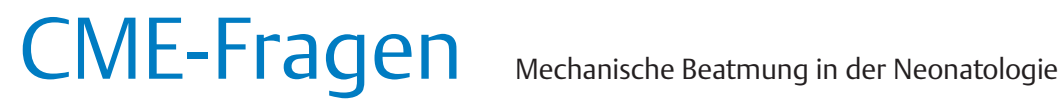

\section{7}

Welche der folgenden Maßnahmen erhöht den mittleren Atemwegsdruck nicht?
A erhöhter positiver endexspiratorischer Druck
B erhöhter inspiratorischer Spitzendruck
C verlängerte Inspirationszeit
D erhöhte Beatmungsfrequenz
E verlängerte Exspirationszeit

8

Welcher Effekt wurde in Studien zur permissiven Hyperkapnie nachgewiesen?
A niedrigere BPD-Rate
B niedrigere Pneumothoraxrate
C höhere Hirnblutungsrate
D höherer $\mathrm{FiO}_{2}$-Bedarf
E niedrigere Infektionsrate

Die Volumengarantiebeatmung
A ist eine Form der volumenkontrollierten Beatmung.
B erfordert vom Anwender, das inspiratorische Tidalvolumen vorzugeben.
C erfordert ein waches, gut mitarbeitendes Kind.
D ist eine drucklimitierte Beatmung mit variablem Inspirationsdruck.
E kann unabhängig von Tubuslecks eingesetzt werden.

Die folgenden Faktoren können mit beatmungsbedingten Lungenschäden assoziiert sein. Welcher ist der wichtigste?
A Inspirationsdruck
B Bias Flow
C $\mathrm{FiO}_{2}$
D Frequenz
E Tidalvolumen 